\title{
Implementation of HEMP Waveforms for Time-Domain Modeling
}

\author{
Derek Mallinson \\ Laboratory of Electromagnetic Systems Engineering \\ Cranfield University \\ Shrivenham, UK \\ d.mallinson@cranfield.ac.uk
}

\author{
Ivor Morrow \\ Laboratory of Electromagnetic Systems Engineering \\ Cranfield University \\ Shrivenham, UK \\ i.1.morrow@cranfield.ac.uk
}

\begin{abstract}
This paper presents recent advances in the development of HEMP waveforms with causal responses suitable for implementation in Finite Difference Time Domain models. New time-domain implementations of the HEMP environment are proposed using direct and indirect employment of the HEMP waveform. Numerical instabilities of the waveform are addressed and a new stable waveform matched to the IEC standard has been evaluated. It is concluded that the implementation of the quotient exponential model provides a stable and accurate waveform for time-domain modelling.

Index Terms-HEMP, Electromagnetic Pulse, FDTD, Time Domain, Double Exponential, DEXP, QEXP, Quotient Exponential.
\end{abstract}

\section{INTRODUCTION}

Recent events have renewed interest in the susceptibility of electronics and electromagnetic systems to High-Altitude Electromagnetic Pulses (HEMP). The high power and lowfrequency characteristics of HEMP waveforms increase the difficulty in simulating the effects of a HEMP in real-world environments. Consequently, little research effort has been directed at HEMP numerical waveform modelling over the last 30 years. The advent of accessible high-performance computing and improved time-domain modelling techniques has improved the opportunity to conduct accurate and efficient modelling of EMP waveforms in large-scale scenarios.

\section{HEMP WAVEFORMS}

The HEMP standard environment is described using three waveforms, the early-time (E1), intermediate-time (E2) and late-time (E3) pulses. Together these represent the entirety of the HEMP environment. The extant HEMP standard utilises a Double Exponential (DEXP) waveform that suffers from temporal instability. An alternative waveform using Quotient Exponentials (QEXP) has been developed which provides a stable alternative to the IEC standard when implemented directly in numerical simulations. Similarly, the use of a matched Gaussian can provide accurate pulse characteristics in a simplified model.

\section{A. Evaluating the IEC 61000 Waveform}

The current IEC standard for the description of the HEMP environment utilises a double exponential (DEXP) waveform 978-1-5386-5204-6/18/\$31.00 @2018IEEE
[1]. Both the temporal and spectral waveforms are described in 1 .

$$
\begin{gathered}
E_{\mathrm{D}}(t)=E_{0} k\left(e^{-\alpha\left(t-t_{0}\right)}-e^{-\beta\left(t-t_{0}\right)}\right) u\left(t-t_{0}\right) \\
E_{\mathrm{D}}(\omega)=E_{0} k\left((j \omega+\alpha)^{-1}-(j \omega+\beta)^{-1}\right) e^{-j \omega t_{0}}
\end{gathered}
$$

Where $E_{0}$ is the peak E-field strength $(\mathrm{V} / \mathrm{m}), t$ is the time (s), $t_{0}$ is the time delay (s), $\alpha$ and $\beta$ are the pulse decay and risetime constants respectively $(\mathrm{rad} / \mathrm{s}), k$ is a normalisation factor, $\omega$ is the radial frequency $(\mathrm{rad} / \mathrm{s})$ and $u\left(t-t_{0}\right)$ is a Heaviside step function that "switches on" the waveform at $t=t_{0}$. The stability of the time-domain waveform can be evaluated using Taylor's theorem such that if the second-order derivative is continuous the waveform is stable [2]. An unstable waveform is likely to instigate small errors in the model resulting in an oscillating E-field response that increases exponentially. The second-order derivative of the DEXP waveform can be shown to be discontinuous due to the presence of the Heaviside stepfunction as shown in 2 .

$$
\frac{\mathrm{d}^{2}}{\mathrm{~d} t^{2}}(u(t))=\frac{\mathrm{d}}{\mathrm{d} t}(\delta(t))=\text { undefined }
$$

When the Heaviside function is removed, the DEXP is shown to be stable, as shown in 3 .

$$
\begin{aligned}
& \frac{\mathrm{d}^{2}}{\mathrm{~d} t^{2}}\left(E_{0} k\left(e^{-\alpha\left(t-t_{0}\right)}-e^{-\beta\left(t-t_{0}\right)}\right)\right) \\
& =E_{0} k\left(\alpha^{2} e^{-\alpha\left(t-t_{0}\right)}-\beta^{2} e^{-\beta\left(t-t_{0}\right)}\right)
\end{aligned}
$$

The DEXP withour Heaviside step-function is, however, quixotic and rapidly tends to $-\infty$ before $t=t_{0}$. The DEXP waveform is therefore not implementable in Time-Domain models.

\section{B. New HEMP Proposed Waveform}

An alternative HEMP environment, as proposed by Baum [3], uses a quotient exponential (QEXP) waveform as described in 4 .

$$
\begin{aligned}
E_{\mathrm{Q}}(t) & =\frac{E_{0} k u\left(t-t_{0}\right)}{e^{\beta\left(t-t_{0}\right)}+e^{-\alpha\left(t-t_{0}\right)}} \\
E_{\mathrm{Q}}(\omega) & =\frac{E_{0} k \pi}{\alpha+\beta} \operatorname{cosec}\left(\pi \frac{j \omega+\beta}{\alpha+\beta}\right) e^{-j \omega t_{0}} .
\end{aligned}
$$


Again this utilises a Heaviside step-function, however, when this is removed, the waveform rapidly tends to 0 before $t=t_{0}$. Further the waveform is continuous in its second derivative, as shown in 5 .

$$
\begin{array}{r}
\frac{\mathrm{d}^{2}}{\mathrm{~d} t^{2}}\left(\frac{E_{0} k}{e^{\beta\left(t-t_{0}\right)}+e^{-\alpha\left(t-t_{0}\right)}}\right) \\
=E_{0} k\left(\frac{2\left(\alpha e^{-\alpha\left(t-t_{0}\right)}-\beta e^{\beta\left(t-t_{0}\right)}\right)^{2}}{\left(e^{-\alpha\left(t-t_{0}\right)}+e^{\beta\left(t-t_{0}\right)}\right)^{3}}\right. \\
\left.-\frac{\alpha^{2} e^{\alpha\left(t-t_{0}\right)}+\beta^{2} e^{\beta\left(t-t_{0}\right)}}{\left(e^{-\alpha\left(t-t_{0}\right)}+e^{\beta\left(t-t_{0}\right)}\right)^{2}}\right)
\end{array}
$$

Two QEXP waveforms have been previously proposed by Baum and Leuthauser. Neither of these HEMP environments include E2 or E3 waveforms and the waveforms themselves have significantly different profiles to the IEC standard as shown in Fig. 1. The QEXP waveform can be fitted to the

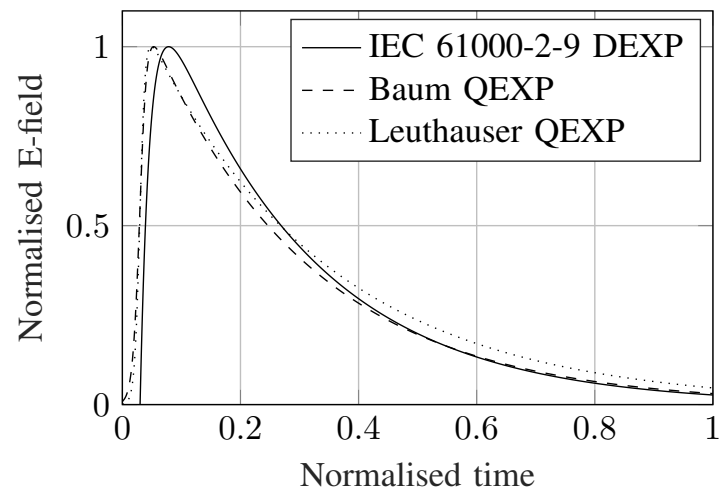

Fig. 1. Normalised IEC DEXP, Baum QEXP and Leuthauser QEXP HEMP waveforms.

IEC waveform using a simple Gauss-Newton Least-Square Errors (LSE) regression across the time domain. The waveform can be simplified into a function of the decay and rise-time coefficients $\left(\alpha\right.$ and $\beta$ ) and time $(t) . E_{0}$ is a constant and can be removed from the regression analysis, $k$ is a normalisation factor raising the peak to 1 . This can be calculated from the rise-time and decay coefficients as shown in 6.

$$
k=e^{\beta t_{\text {peak }}}+e^{\alpha t_{\text {peak }}}
$$

Where, $t_{\text {peak }}$ is the peak time of the pulse, and can be calculated from the rise-time and decay coeffcients as shown in 7

$$
t_{\text {peak }}=\frac{\ln |\alpha / \beta|}{\alpha+\beta}
$$

The QEXP function can therefore be rewritten as shown in 8

$$
E_{Q}(\alpha, \beta, t)_{\text {norm }}=\frac{e^{\beta \frac{\ln |\alpha / \beta|}{\alpha+\beta}}+e^{-\alpha \frac{\ln |\alpha / \beta|}{\alpha+\beta}}}{e^{\beta t}+e^{-\alpha t}}
$$

This can be implemented in a Gauss-Newton LSE regression with 10,000 time steps to fit all three DEXP HEMP waveforms, shown in Fig. 2 and Fig. 3. This new QEXP waveform is directly implementable in Time-Domain models and is matched to represent the DEXP waveform.

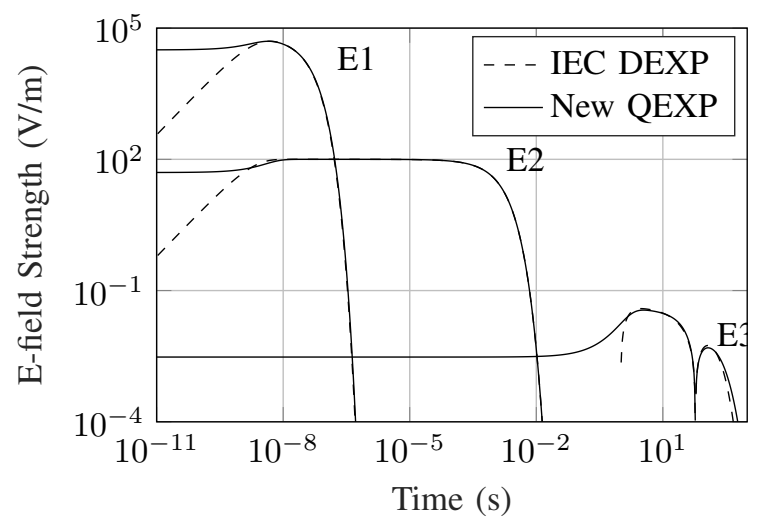

Fig. 2. IEC 61000-2-9 DEXP HEMP and proposed matched QEXP HEMP model in the time domain

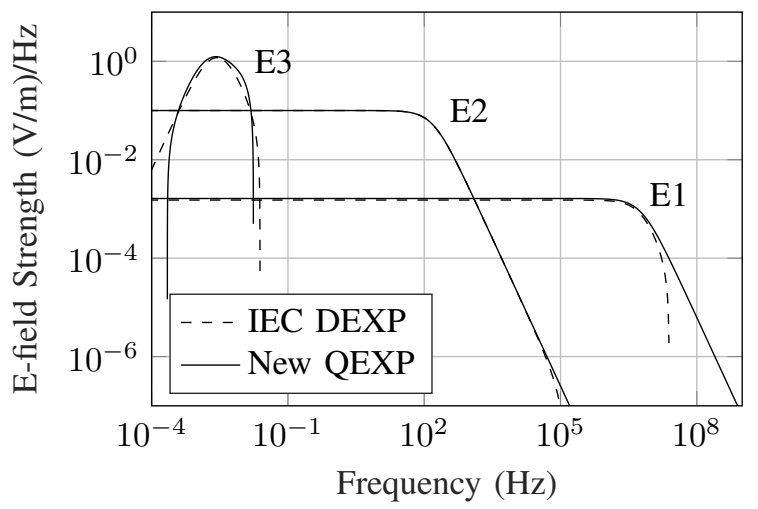

Fig. 3. IEC 61000-2-9 DEXP HEMP and proposed matched QEXP HEMP model in the frequency domain.

\section{Matched Gaussian Waveform}

An alternative to direct implementation of a HEMP waveform, is to use a matched Gaussian pulse. For this analysis the Gaussian pulse is matched in the time domain for implementation in the model, after which the frequency response is convolved with a matching function to reconstruct the DEXP waveform. The Gaussian can be matched to the pulse width, peak time and peak-field as shown in 9.

$$
E_{g}(t)=E_{0} \cdot e^{\frac{-\left(t-\left(t_{p}+t_{0}\right)\right)^{2}}{t_{1}^{2}}}
$$

Where $t_{1}$ is the pulse-width of the DEXP waveform, $t_{p}$ is the peak time of the DEXP waveform and $E_{0}$ is the peak E-field strength of the DEXP waveform. The matched Gaussian is shown in Fig. 4. The Fourier transform can be calculated as shown in 10 .

$$
E_{G}(\omega)=E_{0} t_{1} \sqrt{\pi} e^{-\omega^{2} t_{1}^{2} / 4} e^{-j \omega\left(t_{0}+t_{p}\right)}
$$

The DEXP waveform can be reconstructed from the Gaussian using a matching function $M$ as described in 11 .

$$
M(\omega)=\frac{E_{D}(\omega)}{E_{G}(\omega)}
$$




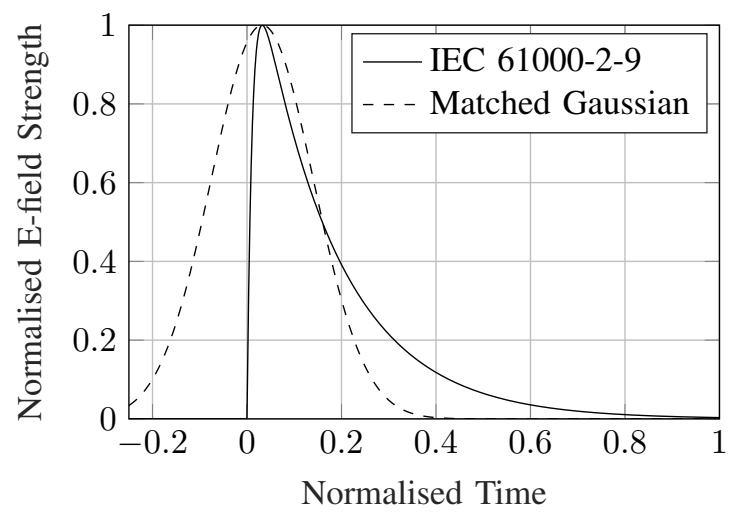

Fig. 4. IEC 61000-2-9 DEXP HEMP and matched Gaussian (time domain).

This is inferred from the Matched Gaussian and DEXP waveform as shown in 12 .

$$
M(\omega)=\frac{k(\beta-\alpha)}{t_{1} \sqrt{\pi}} \cdot \frac{e^{\frac{\omega^{2} t_{1}^{2}+j 4 \omega t_{p}}{4}}}{(j \omega+\alpha)(j \omega+\beta)}
$$

The matched Gaussian and matching function is shown in Fig. 5 .

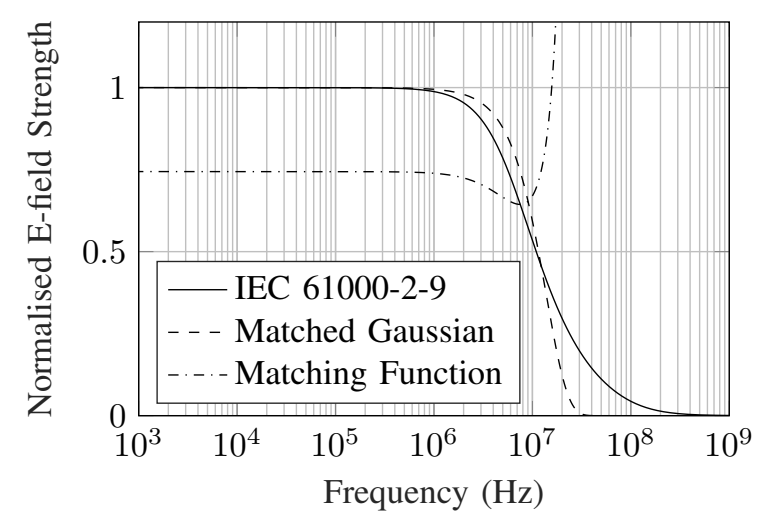

Fig. 5. IEC 61000-2-9 DEXP HEMP, matched Gaussian and Matching Function.

\section{WAVEFORM IMPLEMENTATION AND EVALUATION}

The above waveforms have been implemented in two propagation scenarios; a homogenous dielectric with low conductivity simulating a lossless propagation and a lossy material with complex permittivity. The FDTD numerical models employ an 8-layered Berenger Perfectly Matched Layer (PML) absorber described by Hagness [2].

\section{A. Lossless Material Model}

The lossless material properties and model characteristics are detailed in table III-A.

The expected frequency response is shown in Fig. 6. Implementing the simulation using a Finite Difference Time Domain (FDTD) with maximum frequency $10 \mathrm{GHz}$, the temporal response (shifted in time for presentation) is shown in Fig. 7. This demonstrates that both the Gaussian and QEXP wave-
TABLE I

LOSSLESS MATERIAL SIMULATION PARAMETERS.

\begin{tabular}{|l|l|}
\hline Property & Value \\
\hline Permittivity $\left(\varepsilon_{r}\right)$ & 88 \\
\hline Permeability $\left(\mu_{r}\right)$ & 1 \\
\hline Conductivity $(\sigma)$ & $10 \times 10^{-6} \mathrm{~W}^{-1} m^{-1}$ \\
\hline Length $(L)$ & $100 \mathrm{~m}$ \\
\hline Maximum Frequency $\left(f_{\max }\right)$ & $10 \mathrm{GHz}$ \\
\hline
\end{tabular}

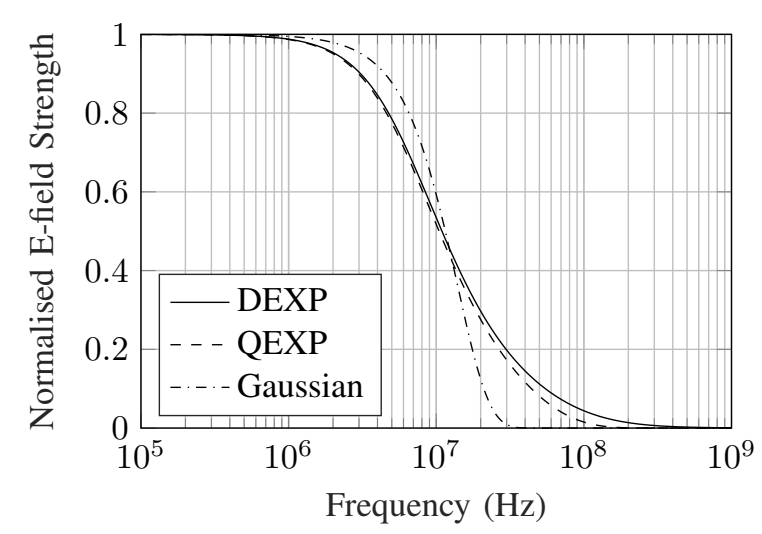

Fig. 6. Frequency response through lossless material.

forms are stable in the simulation. The DEXP waveform suffers dampened oscillations throughout it's temporal response, artificats of the discontinuous DEXP waveform. The DEXP waveform is therefore discounted as an unrealistic implementation of the HEMP environment. The spectral response is calculated using the Fourier Transform of the temporal output from the simulation. The DEXP waveform can be reconstituted from the Gaussian using the matching function as described earlier. These spectral waveforms are shown in Figure 8. This shows good agreement between the Gaussian and QEXP waveform. The reconsituted DEXP suffers from exponential error after $10 \mathrm{kHz}$ most likely caused by the errors induced in numerical approximation of the Gaussian waveform. This may be mitigated against by reducing the Gaussian pulse width, however the errors will likely still be induced at a higher frequency, requiring windowing to eliminate the highfrequency errors.

\section{B. Lossy Material Model}

The lossy material properties and model characteristics are detailed in table III-B. A similar temporal response is seen

\begin{tabular}{|l|l|}
\hline Property & Value \\
\hline Permittivity $\left(\varepsilon_{r}\right)$ & $88+1 i$ \\
\hline Permeability $\left(\mu_{r}\right)$ & 1 \\
\hline Conductivity $(\sigma)$ & $10 \times 10^{-6} \mathrm{~W}^{-1} m^{-1}$ \\
\hline Length $(L)$ & $2 \mathrm{~m}$ \\
\hline Maximum Frequency $\left(f_{\max }\right)$ & $20 \mathrm{GHz}$ \\
\hline
\end{tabular}

in the DEXP waveform with oscillations occuring due to the inherent instability of the pulse. The QEXP waveform shows greater stability and should provide higher accuracy in the higher-frequency spectral response. The temporal output of the 


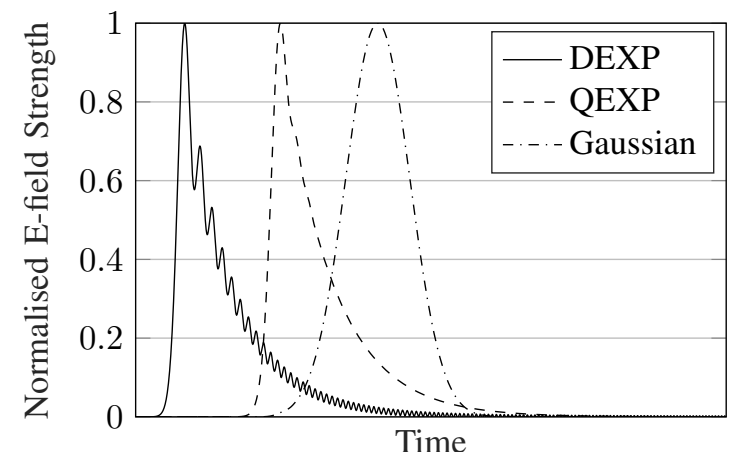

Fig. 7. FDTD Temporal Response of HEMP E1 Waveforms.

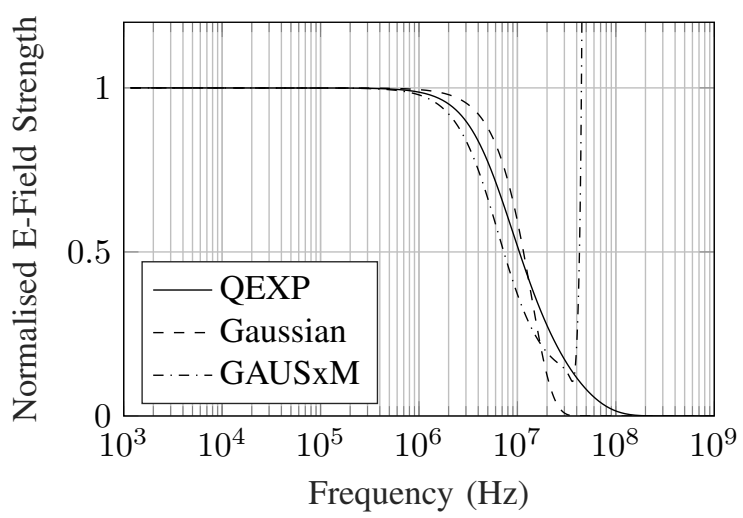

Fig. 8. FDTD Spectral Response of HEMP E1 Waveforms.

FDTD model is shown in Figure 9. A simple analytic model using identical parameters for the paropagation path material has been used to validate the spectral response of the FDTD waveforms.

The difference in E-field responses is shown across the frequency domain and similar errors are seen in both DEXP and QEXP waveform with the exception of an increased difference between analytic and FDTD responses seen in the DEXP waveform above $100 \mathrm{kHz}$. This shows a noticeable improvement in accuracy of directly injected HEMP waveforms using the QEXP expression. The spectral response is shown in Fig. 10. The majority of the discrepency between FDTD and analytic results can be attributed to the limitations in the implementation of FDTD using low-performance processing, limitations in the granularity of the temporal and spacial grids increase the high frequency error and limitations in the domain length increase the low frequency error. However the small difference between QEXP and DEXP accuracies above $100 \mathrm{kHz}$ show the QEXP waveform to be more practical and implementable in time-domain models. Improvements in the FDTD model will show greater discrepency between QEXP and DEXP waveforms and as such the QEXP will be significantly more accurate in high-performance implementations of FDTD.

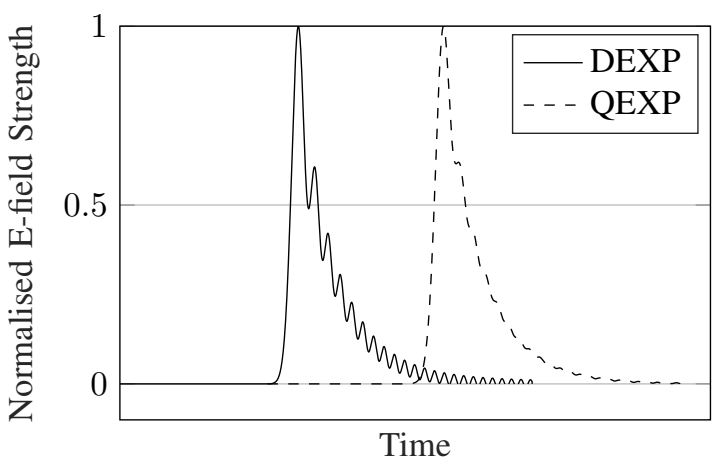

Fig. 9. FDTD Temporal Response of HEMP E1 Waveforms Through Lossy Material.

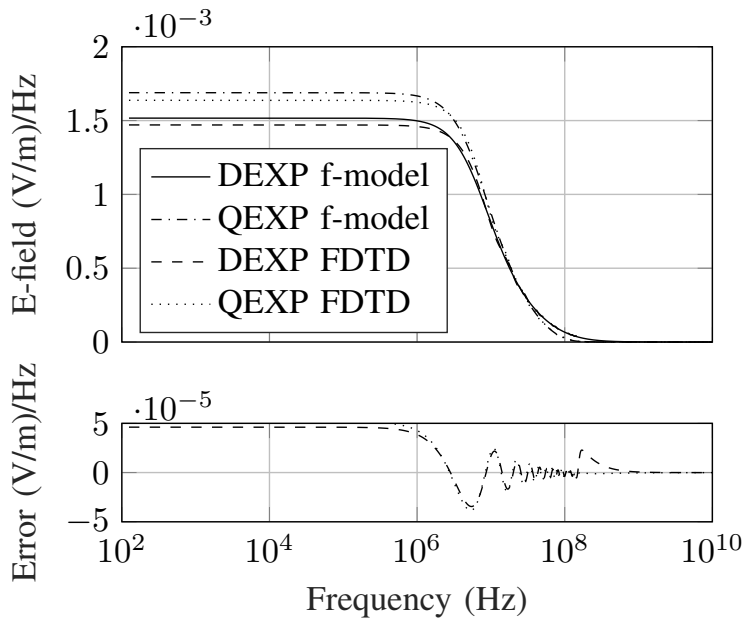

Fig. 10. FDTD Spectral Response of HEMP E1 Waveforms Through Lossy Material with Error.

\section{CONCLUSION}

Three matched HEMP waveforms have been compared using different mathematical expressions. The QEXP matched waveform shows the greatest stability and highest accuracy when implemented in time-domain models. Limitations in the implementation of the Finite Difference Time Domain model limit the improvemen seen using a QEXP waveform, however this waveform will show significantly higher accuracy when implemented in large-scale models parallelised on highperformance processing arrays.

\section{REFERENCES}

[1] CENELEC, "BS EN 61000-2-9:1996, Electromagnetic compatibility (EMC) Part 2: Environment Section 9: Description of HEMP environment - Radiated disturbance - Basic EMC Publication," British Standards Institute, 1996, p. 7 - 15

[2] A. Taflove, "Computational Electrodynamics: The Finite-Difference Time- Domain Method," Artech House, Inc, Chapter 4, 1995

[3] C. E. Baum, "From the electromagnetic pulse to high-power electromagnetics," IEEE Vol.80 No.6, 1992.

[4] K. D. Leuthauser, "A complete EMP environment generated by highaltitude nuclear bursts: data and standardization," Air Force Phillips Laboratory, 1994, theoretical note 364. 